\title{
World Flora Online (WFO) - Quality control workflow for an evolving taxonomic backbone
}

\author{
Raoul Palese ${ }^{\ddagger}$, Cyril Boillat ${ }^{\ddagger}$, Pierre-André Loizeau ${ }^{\ddagger}$ \\ ‡ Conservatoire et Jardin botaniques de la Ville de Genève, Geneva, Switzerland
}

Corresponding author: Raoul Palese (raoul.palese@ville-ge.ch), Cyril Boillat (cyril.boillat@ville-ge.ch), Pierre-André Loizeau (pierre-andre.loizeau@ville-ge.ch)

Received: 09 Apr 2019 | Published: 10 Jul 2019

Citation: Palese R, Boillat C, Loizeau P-A (2019) World Flora Online (WFO) - Quality control workflow for an evolving taxonomic backbone. Biodiversity Information Science and Standards 3: e35307.

https://doi.org/10.3897/biss.3.35307

\begin{abstract}
Initially populated with information gathered from The Plant List, the WFO's taxonomic backbone will be augmented and modernized by newer taxonomic sources delivered by global plant Taxonomic Expert Networks (TENs). At the same time, descriptive data from floras, such as text descriptions, images, geographic distributions, keys, and trait data will be linked to this evolving taxonomic backbone. Semi-automated workflows have to be built to efficiently pull together the work of the TENs and digital floras and monographs into a single source: the WFO portal.

These semi-automated workflows will have to ensure multiple functionalities: format check of the data submitted by the different backbone data providers (TENs or descriptive data providers), integrity and quality checks of the data provided, comparison of these data with the current WFO's taxonomic backbone (matching with the WFO ID's and with the names, check at the level of the family and the taxonomic status attribution, etc.). Applications will also be developed as Decision Making Helper for the TENs: thanks to these applications, TENs will be able to make decisions concerning the resolution of names not present in the current backbone or on the questions of conflicts at the level of the belonging to a family or the taxonomic status for a proposed name.
\end{abstract}


At the end of these processes, new versions of the taxonomic backbone will regularly be delivered to be integrated into the WFO portal.

\section{Keywords}

World Flora Online (WFO), Taxonomic backbone, Quality control workflow

\section{Presenting author}

\section{Raoul Palese}

